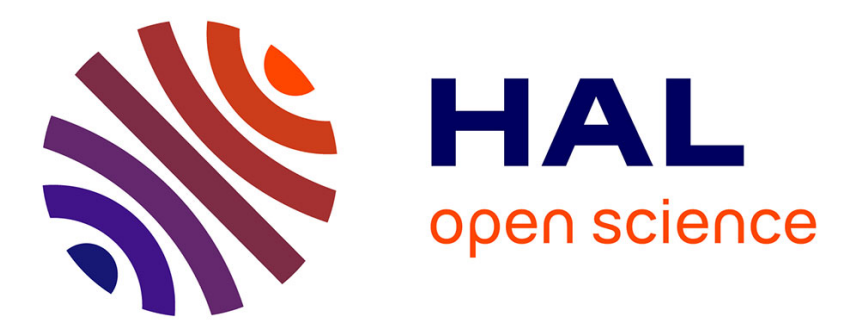

\title{
Optimized simulation architecture for multimodal radar modeling: Application to automotive driving assistance system
}

Steve Pechberti, Dominique Gruyer, Vincent Vigneron

\section{- To cite this version:}

Steve Pechberti, Dominique Gruyer, Vincent Vigneron. Optimized simulation architecture for multimodal radar modeling: Application to automotive driving assistance system. 16th International IEEE Conference on Intelligent Transportation Systems: Intelligent Transportation Systems for All Modes (ITSC 2013), Oct 2013, The Hague, Netherlands. pp.1119-1124, 10.1109/ITSC.2013.6728382 . hal-01059948

\section{HAL Id: hal-01059948 \\ https://hal.science/hal-01059948}

Submitted on 12 Jul 2021

HAL is a multi-disciplinary open access archive for the deposit and dissemination of scientific research documents, whether they are published or not. The documents may come from teaching and research institutions in France or abroad, or from public or private research centers.
L'archive ouverte pluridisciplinaire HAL, est destinée au dépôt et à la diffusion de documents scientifiques de niveau recherche, publiés ou non, émanant des établissements d'enseignement et de recherche français ou étrangers, des laboratoires publics ou privés. 


\title{
Optimized simulation architecture for multimodal radar modeling: Application to automotive driving assistance system
}

\author{
Steve PECHBERTI ${ }^{1}$, Dominique GRUYER ${ }^{2}$ and Vincent VIGNERON ${ }^{3}$
}

\begin{abstract}
This paper presents a new radar sensor modeling for Advanced Driver Assistance Systems (ADAS) prototyping. This virtual model is embedded in the SiVIC platform dedicated to the simulation of vehicles, infrastructure and sensors. Lots of simulators already exist addressing radar simulation, but none of them is designed to address in the same time the following objectives: real-time computation, highly sampled signal generation, multiple radar technologies, and, hardware and software platform integration for ADAS prototyping.

In this paper, we proposed such a solution solving this issue and a focus will be done on the radar sensor modeling, from material modeling to implemented technology and associated signal processing. First results of this radar sensor modeling is carried out in motorway scenario.
\end{abstract}

\section{INTRODUCTION}

Radars are used nowadays in a wide range of application. Whether in aviation to locate aircrafts or in meteorology for the detection of cloud masses. Radar technologies are entered in our daily.

Since more than one decade, more and more driving assistance system are embedded in vehicles in order to improve either the comfort or the safety of the driver. All these new systems require information provided by embedded sensors. Two kinds of sensors exist: proprioceptive ones that provide information about the vehicle dynamics (speed, heading, ...) and exteroceptive ones providing information on the surrounding ego-vehicle environment. Among the more commonly used sensors, the radar is the one strongly dedicated to the ACC and Stop\&Go safety applications. In order to ensure a good inter distance regulation, data coming from radar need to be reliable and robust enough. So it seems to be important to have the capability and the tools to prototype this type of ADAS and identify the use limitations.

Unfortunately, the simulation of this type of sensor is complex, time consuming and often dedicated to a specific domain. Effectively, the term 'radar' is used to described a wide variety of technologies (frequency signal shapes, modulation employed, antenna pattern, ...). Each of them has advantages and disadvantages depending on the task to perform. As for the radar zoology, in the automotive domain,

${ }^{1} \mathrm{~S}$. PECHBERTI is a $\mathrm{PhD}$ student at Laboratoire sur les Interactions Vhicule Infrastructure Conducteur (LIVIC), IFSTTAR, 14, Route de la minire, 78000 Versailles, France - steve.pechberti at ifsttar.fr

${ }^{2}$ D. GRUYER is a researcher at Laboratoire sur les Interactions Vhicule Infrastructure Conducteur (LIVIC), IFSTTAR, 14, Route de la minire, 78000 Versailles, France - dominique.gruyer at ifsttar.fr

${ }^{3} \mathrm{~V}$. VIGNERON is an associated professor at Informatique, Biologie Intgrative et Systmes Complexes (IBISC), UEVE, 40, Rue du Pelvoux, 91020 Evry-Courcouronnes, France - vincent.vigneron at iup.univ-evry.fr radars can used two range frequency $(24 \mathrm{GHz}, 77 \mathrm{GHz})$ with different modulation shapes. These two parameters will defined the range, the field of view, and the accuracy of the embedded sensor.

For these automotive applications, several simulation plaftorms already exist. Among them, we can quote the WinPROP[1] software, which is more dedicated to the propagation issue, PreScan[3] or SE-Workbench-AD[2]. But in all cases, either these softwares are dedicated to propagation channel modeling or to antenna and signal modeling. In few cases, the simulation software implements both aspects, but do not allow real-time computation.

In this paper, we propose a new and innovative optimized architecture of generic automotive radar modeling in order to prototype ADAS in virtual and real-time environment. In this modeling, both antenna and signal modeling, and propagation channel are implemented. In this paper, only the first part is presented with three types of wave modulations for long range configuration $(77 \mathrm{GHz})$, a previous paper with a generic presentation is available in this previous paper[13]. Nevertheless, this modeling can be easily tuned in order to test short-range radar category. Moreover, the modular integration of our modeling in the SiVIC platform allows to apply a large set of radar configurations: radars on one or several car, several radars in same time in order to build not only perception application but also cooperative one, hybrid radar (mixed sensor with short range and long range detection).

In the next section, we will present the real radar components and the signal lifecycle. This part allows to identified the different subparts (hardware components) we need to model in our radar simulation. From the conclusion of the last section, we will propose a set of signal modulations going from signal generation to signal processing (target extractions). Finally, a motorway scenario will be applied to demonstrate the efficiency of this modeling. The results will be shown for the three followning waves modulations: the Frequency Shift Key (FSK) with Two Layers, the Frequency Modulated Continuous Wave (FM/CW) with a SawTooth, and the FMCW with a Triangular pattern.

A conclusion will be provided and some future works will be proposed.

\section{Model Presentation}

To model a radar, we must take into account different aspects of the reality from the modulated signal in an electronic device to the wave propagation through the environment. 
This section presents the different hypothesis and principles implemented in the presented radar model.

\section{A. Radar component modeling}

A radar antenna is composed of module units having particular task to operate in the complete system. The figure below (Fig. 1) detailed the various components included in the model presented here. In blue are represented the elements involved in the generation and the reception of the radar signal. Represented in green are items where the signal travels through the environment in the form of electromagnetic wave. Finally in black is represented the processing unit for extracting information from the received signal.

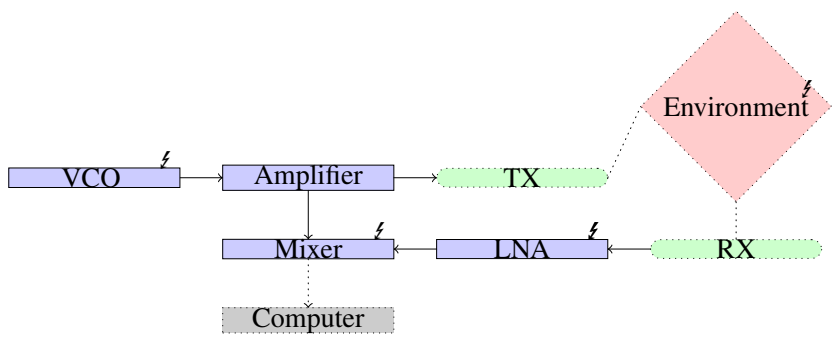

Fig. 1. Hardware components overview

The chain of transmission / reception consists of a Voltage Control Oscilloscope (VCO) that generates the modulation of the carrier frequency, an Amplifier that redefines the transmission power, a Low Noise Amplifier (LNA) that allows a better ratio for $\frac{\text { Signal }}{\text { Noise }}$ and a Mixer that sums all contributions returned by the environment with the transmitted signal.

The chain of propagation implies model data from the transmitter (TX) and the receiver (RX) and computed returned path from propagation channel implemented in the SiVIC platform.

Finally, the computation chain is only composed by the computer, where signal processing are defined.

1) Hardware imperfection: Because all materials are not perfect, a noise model is also defined for these hardware, such as thermal noise and clutter noise. All noises taken into account are considered as white or pink noises.

a) The components noise: is due to imperfection on hardware. Each component of the antenna is caracterised with a noise and gain factor. Noise factor for components of the channel set is defined, according to Friss formula, by: $F_{\text {hard }}=F_{V C O}+\frac{F_{L N A}-1}{G_{V C O}}+\frac{F_{M i x e r}-1}{G_{V C O} G_{L N A}}$ with, respectively $F_{i}$ and $G_{i}$ the noise factor and the gain factor for the component $i$.

b) Coupling effect: results in the fact that emitter and receiver are closed and work with high frequencies. The coupling between antenna is characterized by an attenuation of the signal, a Doppler shift frequency and a signal delay. The coupling between antenna is included in the reconstruction of the signal received in the form:

$$
s_{r}^{(*)}(t)=G_{\text {coup }} \cdot e^{\left(2 \pi i f_{d_{\text {coup }}} t\right)} \cdot e^{\left(2 \pi i F_{c} \tau_{\text {coup }}\right)} * s_{t}(t)
$$

with, $G_{\text {coup }}$ the attenuation factor, $f_{d_{\text {coup }}}$ the doppler shift associated to coupling, $\tau_{\text {coup }}$ the associated delay.

All these representations are about the hardware and signal disturbance inside the antenna, the representation of the radar antenna for propagation inside the virtual environment is defined as an isotropic radiator. However, unlike model, radar antenna does not emit uniformly in all directions.

2) Antenna diagram: To get closer to the emission of a real antenna a diagram defines the antenna gain as a function of the emission angle. Different representations are availables, from well-known pattern to pre-recorded tables. Although the model is evolving in a $3 \mathrm{D}$ environment, it is possible to use antenna diagram defined in $2 \mathrm{D}$, it is then considered that the transmission is mainly in the horizontal plane of the antenna.

\section{B. Signal lifecycle modeling}

Generation $\rightarrow$ Propagation $\rightarrow$ Reception $\rightarrow$ Computation

Fig. 2. Signal lifecycle

Simulate a radar signal lifecycle requires at least four steps:

- Generate the signal to be transmitted

- Propagate the signal in the environment

- Build the signal back to the antenna from echos

- Extract information about targets

\section{1) Signal Generation:}

Radar signal generation relies on analytical forms modulations encountered.

In automotive domain, employed radars are commonly from the Continuous Wave (CW) family. So the basic form of the generated signal is usually defined as

$$
s_{t}(t)=A \cdot \operatorname{Cos}\left(2 \pi \int_{0}^{t} f_{\bmod }(\tau) d \tau\right)
$$

For convenience in computation, the complex form is employed

$$
s_{t}(t)=A \cdot e^{\left(2 \pi i \int_{0}^{t} f_{\text {mod }}(\tau) d \tau\right)}
$$

with $f_{\text {mod }}$ depending on the modulation.

The modulation $f_{\text {mod }}$ depends on the technology employed. Some of possible modulations are described below in the section II-A.

2) Received signal computation: For this task, two approaches can be used in order to build the returned signal.

The first one is based on the analytic form of the returned signal. The electromagnetic wave travel space with light celerity, so the delay between the emission of the wave and the return of its echos from the antenna is $t_{d}=\frac{2 d}{c}$. This mean that the received signal at the time $t$ is similar to the transmitted signal at the time $t-t_{d}, s_{r}(t) \approx s_{t}\left(t-t_{d}\right)$. If the electromagnetic wave hits an object with a non zero velocity, the signal is also doppler shifted by $f_{d}=\frac{2 v f_{c}}{c}$. In 
this approach, we must define the analytic received signal forms for all modulations taken into account.

The other approach is based on the use of filters. All induced perturbations can be seen as a filter applied on the transmitted signal.

a) The delayed filter $G_{\phi}$ : is employed to simulate the phase shift of the signal due to the time travel, and it is expressed as:

$$
G_{\phi}(t)=e^{2 \cdot \pi \cdot i \cdot F_{c} \cdot \tau_{d}}
$$

where $\tau_{d}=\frac{2 . R}{c}$ with, $F_{c}$ the carrier frequency, $R$ the distance traveled by the wave, $c$, the light celerity.

b) The doppler filter $G_{d}$ : is associated to the Doppler effect of the wave echos on moving targets, it is expressed as:

$$
G_{d}(t)=e^{2 \cdot \pi \cdot i \cdot f_{d} \cdot t}
$$

where $f_{d}=\sqrt{\frac{c-\Delta v}{c+\Delta v}} F_{c}$ with $c$ is the light velocity and $\Delta v$ the speed difference between the antenna and a mobile entity (carsq, walkers, ...). As we manipulate electromagnetic waves, we use the doppler relativist formula, where emitter and receiver have a symetric function and where only the difference of velocities is efficient.

c) The gain filter $G_{g}$ : applies to the signal the attenuations from medium crossing and surface interactions. It is expressed as:

$$
G_{a}^{(i)}(t)=\operatorname{gain}_{i}
$$

where gain is obtained according to the propagation channel employed.

The received signal is then defined, for a particular path $i$, as:

$$
s_{r}^{(i)}(t)=G_{\phi}^{(i)}(t) * G_{d}^{(i)}(t) * G_{g}^{(i)}(t) * s_{t}(t)
$$

To compute the complete received signal, we just sum all returned contributions to the antenna, and we mix it with the transmitted signal

$$
s_{r}(t)=\left(\sum_{i=1}^{n} s_{r_{i}}^{(i)}(t)\right) * \overline{s_{e}(t)}
$$

3) Signal processing: As modulation is specific to a technology, the signal computation is also associated to the employed technology.

\section{IMPLEMENTATION}

The model is splitted in two main parts. The first one deals with the generoic aspect of the radar sensor and the other one deals with the specific aspects: modulations and antennas configuration.

\section{A. Implemented modulations}

As previously mentioned, there are many technologies for radar or modulations. Of course, most manufacturers define their own modulations often composed of several simple modulations. Modulations defined here cover the various elementary forms of signals used in the detections of targets. About signal processing, a windowing (Hann) is necessary before processing Fourier transformation. After the DFT, the signal is filtered before trying to extract remarquable frequencies (doppler frequency, beat fraquency, ...), among these processes, we can cite gaussian filter.

1) The Frequency Shift Key (FSK) Family: This kind of Radar mainly use the doppler frequency for target detection[14]. This means that a zero velocity difference between the antenna and the target results in a no detection. Thus this model is not intended to equip the vehicles but is however suitable for use wayside.

a) FSK with Two Layers: is a discrete modulation of the carrier frequency. It consists of two levels, one per half period. It is defined by

$$
\begin{aligned}
f_{\text {mod }}(t) & =\left\{\begin{array}{cc}
F_{c} & 0<t<\frac{T}{2} \\
F_{c}+B W & \frac{T}{2}<t<T
\end{array}\right. \\
s_{t}(t) & =\left\{\begin{array}{cc}
A . e^{\left(2 \pi i\left(F_{c}\right) t\right)} & 0<t<\frac{T}{2} \\
A . e^{\left(2 \pi i\left(F_{c}+B W\right) t\right)} & \frac{T}{2}<t<T
\end{array}\right.
\end{aligned}
$$
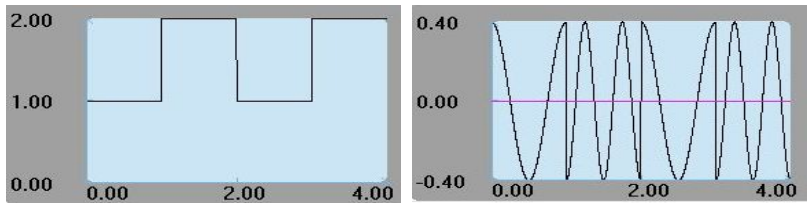

Fig. 3. FSK 2 Layers: Modulation (left), Signal (right)

For the computation, FSK only allows to detect Doppler frequencies. The principle is simple, it retains only a halfsample period (corresponding to a plateau) and then rebuilt in the signals for each level of the modulation. The Fourier transform of the sub-sample is used to determine the relative speed of the target and the phase associated with this frequency allows to determine the distance to the target.

$$
\begin{aligned}
v & =\frac{c \cdot f_{d}}{2 \cdot F_{c}} \\
R & =\frac{c \cdot T \cdot \phi_{f_{d}}}{4 \cdot B W}
\end{aligned}
$$

with $c$ light celerity, $f_{d}$ the doppler frequency, $\phi_{f_{d}}$ the phase difference between the two layers at $f_{d}, F_{c}$ the carrier frequency, $T$ the signal period and $B W$ the bandwith.

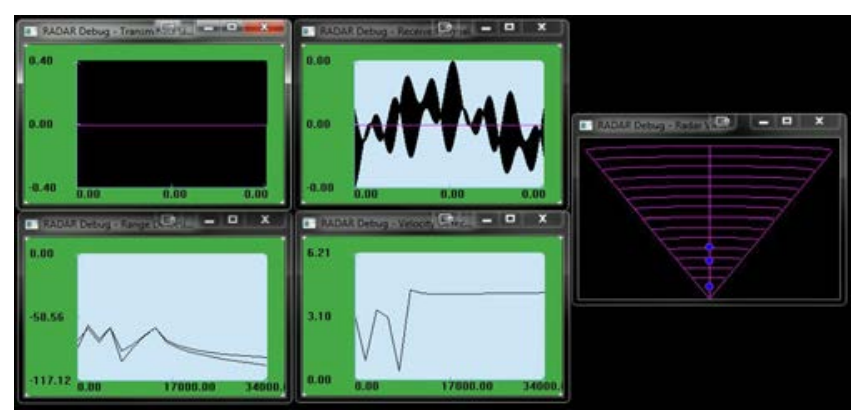

Fig. 4. FSK 2 Layers: Computation Results 
2) FMCW Family: The linear modulation of the signal in time allows the detection of non mobile object by inducing variation on the phase shifting which are detectables adding to the doppler shift also detectable[4].

a) SawTooth Modulation: is only composed of one up sweep.

$$
\begin{aligned}
f_{\text {mod }}(t) & =F_{c}+\frac{B W}{T} t \quad 0<t<T \\
s_{t}(t) & =A \cdot e^{2 \pi i\left(\frac{B W}{2 T} t^{2}+F_{c} t\right)} \quad 0<t<T
\end{aligned}
$$
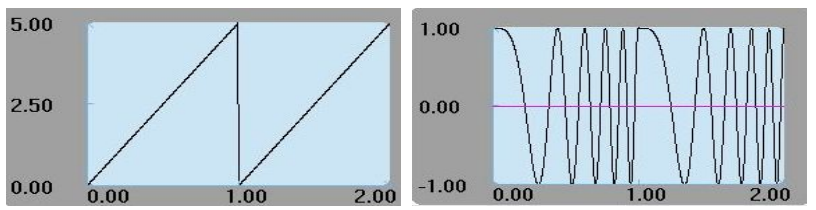

Fig. 5. FMCW SawTooth: Modulation (left), Signal (right)

The associated processing is carried out on a set of $\mathrm{N}$ periods. dual FFT[8][5] is then performed on the signal. The first DFT provides the $f_{b}$ frequency whereas the second provide the $f_{d}$ frequency. The defined plan allows to characterize both the speed and the distance to the target. For moving targets, however, the beat frequency induced by the distance to the target is disrupted by the addition of Doppler component, the distance information is then noisy.

$$
\begin{aligned}
R & =\frac{c \cdot T \cdot f_{b}}{B W} \\
v & =\frac{c \cdot f_{d}}{2 \cdot F_{c}}
\end{aligned}
$$

with $c$ light celerity, $F_{c}$ the carrier frequency, $T$ the signal period, $B W$ the bandwith, $f_{b}$ and $f_{d}$ the beat frequency and the doppler frequency.

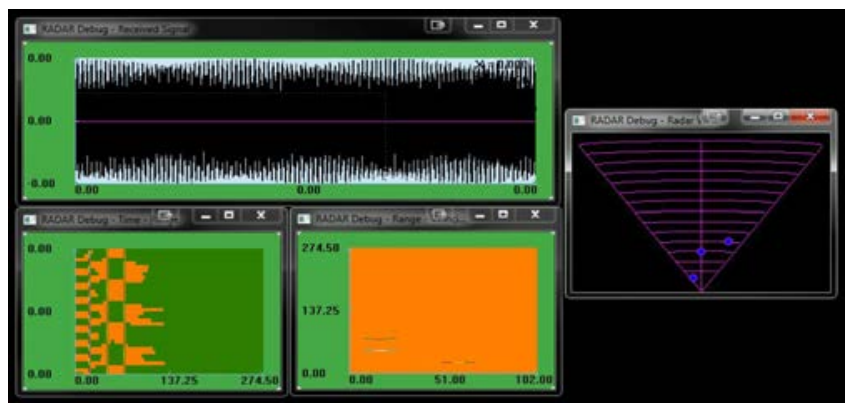

Fig. 6. FMCW SawTooth: Computation Results

b) Triangular Modulation: combines two sweeps[9].

$$
\begin{aligned}
f_{\text {mod }}(t) & =\left\{\begin{array}{cc}
\frac{B W}{T} t+F_{c} & 0<t<\frac{T}{2} \\
-\frac{B W}{T} t+\left(F_{c}+2 B W\right) & \frac{T}{2}<t<T
\end{array}\right. \\
s_{t}(t) & =\left\{\begin{array}{l}
A \cdot e^{2 \pi i\left(\frac{B W}{T} \frac{t^{2}}{2}+F_{c} t\right)} \\
A \cdot e^{2 \pi i\left(-\frac{B W}{T} t^{2}+\left(F_{c}+2 B W\right) t-\frac{B W}{2} T\right)}
\end{array}\right.
\end{aligned}
$$

In the second part of the test, the target moves away ${ }^{\left[t_{2+}, t_{3}\right]}$ $0<t<\frac{T}{2}$ from the radar with a speed of expulsion $\left(v_{\exp }>0\right)$ which $\frac{T}{2}<t<T$ increases with the distance, then it approaches ${ }^{\left[t_{3}, t_{4-}\right]}$ with a
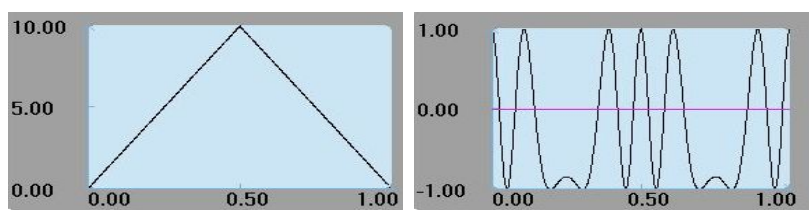

Fig. 7. FMCW Triangular: Modulation (left), Signal (right)

The treatment is carried out over one period of the signal. We can use several period in order to obtain a better average signal. On each of the ramps (uplink and downlink), we determines the beat frequency using a fourier transform[11][6]. It is then determined with these frequencies, the speed and distance to the target.

$$
\begin{aligned}
v & =\frac{c \cdot\left(f_{b-}+f_{b+}\right)}{2 \cdot F_{c}} \\
R & =\frac{c \cdot T \cdot\left(f_{b+}-f_{b-}\right)}{8 \cdot B W}
\end{aligned}
$$

with $c$ light celerity, $F_{c}$ the carrier frequency, $T$ the signal period and $B W$ the bandwith, $f_{b-}$ and $f_{b+}$ the beat frequency on the downsweep and on the upsweep.

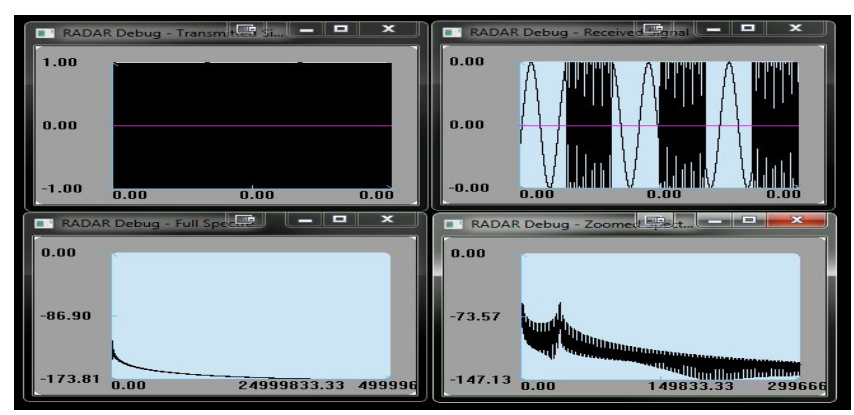

Fig. 8. FMCW Triangular: Compuation results

\section{RESULTS}

This model will evolve in a well detailed environment with high resolution models of the scene (relief, road signs), vehicles, walkers, and so on[15]. So this model must be the more realistic as possible to be substitute to a real sensor in the hardware and software in the loop prototype platform.

\section{A. scenario}

The scenario set up to test the model is a vehicle whose the distance with the radar varies between $r_{\min }$ and $r_{\max }$ and the relative velocity between $v_{\min }$ and $v_{\max }$. Figure 9 shows this evolution.

At first ${ }^{\left[t_{0}, t_{1-}\right]}$, the target moves away of radar at a constant speed $v_{\text {test }}$ from $r_{\min }$ to $r_{\max }$. Then ${ }^{\left[t_{1+}, t_{2-}\right]}$, it gets closer at constant speed $-v_{\text {test }}$.

speed of expulsion that decreases with distance. 


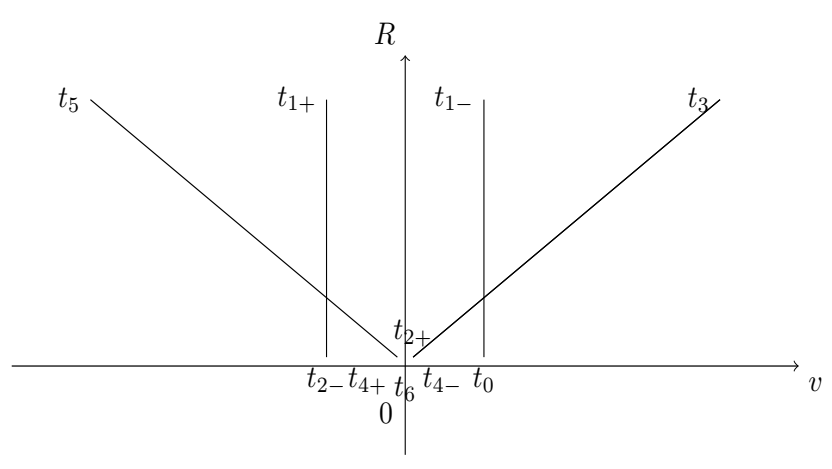

Fig. 9. Range and Velocity evolution during test scenario

In the last part of the test, the target moves away ${ }^{\left[t_{4+}, t_{5}\right]}$ from the radar with a closing speed $\left(v_{c l o}>0\right)$ which increases with the distance, then approaches ${ }^{\left[t_{5}, t_{6}\right]}$ with a closing speed decreases with distance.

This device is used to test the models in areas easily manageable.

The simulation time is 12 seconds with a sample period of $40 \mathrm{~ms}$. Three modulations have been tested in this environment.

\section{B. Reliability}

All modulations presented and tested in this article do not operate in the same value scopes for distance or speed. A short range radar has a range of about $50 \mathrm{~m}$ while for a long range it is about $150 \mathrm{~m}$. In terms of relative speed, the higher the radar sees farther, the more it must be able to perceive high speeds.

1) Short range reliability: Among the tested modulations and parameterizations consistent with the automotive field, the sawtooth modulation is one that best represents this radar category. The close relationship between the period $(T)$ and accuracy in both speed and distance makes for this modulation a small application scope.

Figure I shows the parameters used for the simulation as well as the area in which the model was tested.

\begin{tabular}{|l|l|l|}
\hline FMCW - SawTooth & Carrier Frequency & $77 \mathrm{GHz}$ \\
& Period & $75 \mathrm{~s}$ \\
& BandWidth & $300 \mathrm{MHz}$ \\
& Sample Rate & $20 \mathrm{M} \mathrm{Hz}$ \\
& Generation Length & $100 . \mathrm{T}$ \\
& Range Domain & {$[2.5 \mathrm{~m}, 60 \mathrm{~m}]$} \\
& Speed Domain & {$\left[2 \mathrm{~m} . \mathrm{s}^{-1}, 20 \mathrm{~m} . \mathrm{s}^{-1}\right]$} \\
\hline
\end{tabular}

TABLE I

MOdUlation PARAMETERS FOR RELIABILITY TESTS

Figure 10 shows the errors obtained on measures between the reference and the model output. Average precision for the distance is one meter as for speed.

2) Long range reliability: To represent the category of long range radars, the triangular modulation was used. It offers good precision and scope - tests have shown that up to 300 meters accuracy was correct.
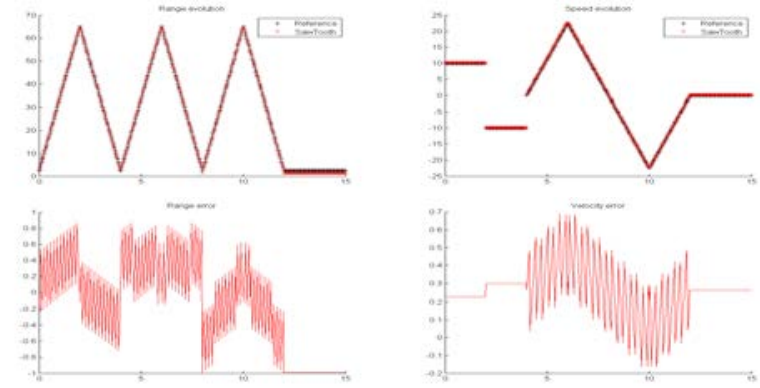

Fig. 10. SawTooth: Range evolution (top left), velocity evolution (top right), range error (bottom left), velocity error (bottom right)

\begin{tabular}{|l|l|l|}
\hline FMCW - Triangular & Carrier Frequency & $77 \mathrm{GHz}$ \\
& Period & $1 \mathrm{~ms}$ \\
& BandWidth & $100 \mathrm{MHz}$ \\
& Sample Rate & $2 \mathrm{MHz}$ \\
& Generation Length & $3 . T$ \\
& Range Domain & {$[2.5 \mathrm{~m}, 300 \mathrm{~m}]$} \\
& Speed Domain & {$\left[2 \mathrm{~m} . \mathrm{s}^{-1}, 80 \mathrm{~m} . \mathrm{s}^{-1}\right]$} \\
\hline
\end{tabular}

TABLE II

MOdULATION PARAMETERS FOR RELIABILITY TESTS
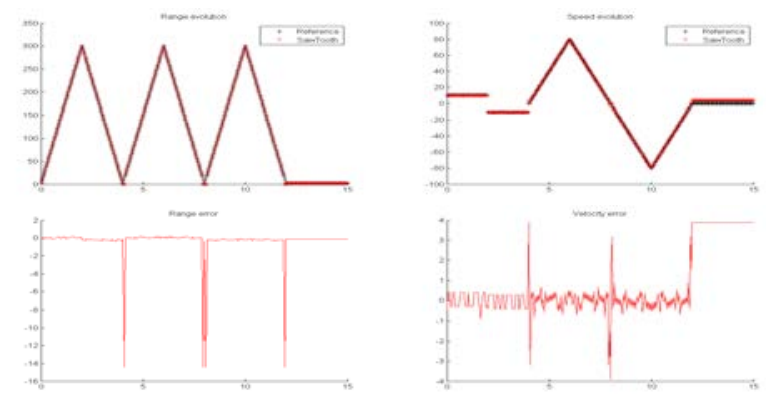

Fig. 11. Triangular: Range evolution (top left), velocity evolution (top right), range error (bottom left), velocity error (bottom right)

3) Special issue: A final modulation tested implementation of this simulator is the FSK with two layers. This modulation has not been selected in this article to be part of one or other of previous categories because of its perfomances. Table III-B.3 present the properties used in this test and Figure 12 shows the results.

\begin{tabular}{|l|l|l|}
\hline FSK - 2 Layers & Carrier Frequency & $77 G H z$ \\
& Period & $0.5 \mathrm{~s}$ \\
& BandWidth & $1 \mathrm{MHz}$ \\
& Sample Rate & $2 \mathrm{MHz}$ \\
& Generation Length & $3000 . \mathrm{T}$ \\
& Range Domain & {$[2.5 \mathrm{~m}, 120 \mathrm{~m}]$} \\
& Speed Domain & {$\left[2 \mathrm{~m} . \mathrm{s}^{-1}, 80 \mathrm{~m} . \mathrm{s}^{-1}\right]$} \\
\hline
\end{tabular}

As can be seen, the higher the speed $(t \in[5.5,6.5]$ and $t \in[9.5,10.5])$, the greater the distance measurement is wrong. However, the measurement of velocity remains. This modulation is more suitable for measuring speed, as speed cameras, as to be embedded in a vehicle for the implementation of system driver assistance.

The model precision is globally consistent with the needs 

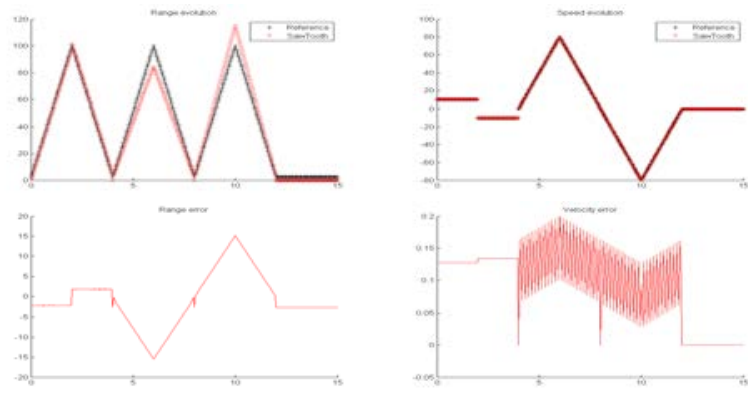

Fig. 12. Two Layers: Range evolution (top left), velocity evolution (top right), range error (bottom left), velocity error (bottom right)

of driving assistance systems namely an accuracy of one meter.

\section{Performances}

Table III shows the average computation time in the simulation loop calculation for the reconstruction of the received signal to extract the information of target. These times were obtained for samples of 375 .

\begin{tabular}{|l|l|l|}
\hline Modulation & \multicolumn{2}{|c|}{ processing time } \\
& average & maximum \\
\hline FSK - 2 Layers & $7 \mathrm{~ms}$ & $37 \mathrm{~ms}$ \\
FMCW - SawTooth & $16 \mathrm{~ms}$ & $62 \mathrm{~ms}$ \\
FMCW - Triangular & $3 \mathrm{~ms}$ & $16 \mathrm{~ms}$ \\
\hline
\end{tabular}

TABLE III

MODULATION PERFORMANCES FOR TARGET DETECTION

The maximum value is obtained during the first iteration when the creation of the various objects and generating the signal to be transmitted.

Current performance of the model are compatible with real-time operation. This performance is mainly due to the size of the useful signal in the treatment of information and number of operations necessary to obtain the information.

\section{SiVIC Integration}

This model has been integrated into the simulation platform SiVIC as a new plug-in. Integration and access to information of the propagation channel are operational and the total time computation including the propagation channel processing is less than $40 \mathrm{~ms}$. In terms of interaction with third-party applications, an output frame defined according to commercial radar frame format has been implemented, it allows via the SiVIC communication bus to replace a real sensor by the presented model.

\section{CONCLUSION}

Radar is a key sensor for the development of embedded driving assistance systems. Some simulation platform of this type of sensor already exist but provide either very complex and unrealistic results with bad time computation, or very simple modeling with a real-time working. In this paper, we have proposed a new and innovative alternative of the existing modeling. This virtual radar modeling is implemented as a new dynamic module in the SiVIC platform dedicated to the vehicle, environment and sensor modeling for ADAS prototyping.

The current performances of this model are compatible with real-time operation in order to be substituted by a real sensor during the different steps of prototyping cycle. To achieve real time computation with high defined propagation channel, only some thousands of returned paths can be used.

The study of these sensors has also revealed the critical role of dedicated processing chipsets. Effectively, without such a system, it is difficult to provide reliable and efficient results in the output of the radar (target distance, velocity, heading, temporal tracking). The adding of a tracking stage will provide reliable information in time and a new set of indicators on target. A previously tracking algorithm[17] specificaly developed for laser scanner processing will be adapted for this model.

Finally, we have proposed a set of three wave modulations dedicated for transportation domain. These modulations have been tested and validated with motorway scenario included one front obstacle which drives on the same lane with an evolutive speed. The results obtain from these different radar configuration confirm that the model is relevant and suitable. In a future work, we will add new interesting modulations[12] in order to complete the functionnalities of this automotive radar modeling.

\section{REFERENCES}

[1] http://www.awe-communications.com/

[2] http://www.oktal-se.fr/

[3] http://www.tass-safe.com/en/home/details/news/50

[4] D. E. Barrick, "FM/CW Radar signals and Digital Processing", in NOAA (ERL 283-WPL 26), 1973.

[5] A. Wojtkiewicz, J. Misiurewicz, M. Nalecz, K.Jedrzejewski, K.Kulpa, "Two-dimensional signal processing in FMCW radars", in Proc. XX KKTOiUE, 1997, pp. 475-480.

[6] A. G. Stove "Linear FMCW radar techniques", in IEE Proceedings F (Radar and Signal Processing), Vol. 139, No. 5, 1992, pp. 343-350.

[7] D. William, "FMCW MMW Radar for Automotive Longitudinal Control", University of California, Berkeley, 1997.

[8] A. Jalil, H. Yousaf, F. Fahim, et al. FMCW Radar Signal Processing Scheme

[9] V. Winkler "Range Doppler Detection for automotive FMCW Radars", in Proceedings of the 4th European Radar Conference, 2007.

[10] M. Wahab, Y. Wijayanto "Signal processing aspects of FM-CW radar", in Proceedings of the 4th European Radar Conference, 2007.

[11] H. Parrish "An Overview of FMCW Systems in MATLAB".

[12] A. Bazzi, T. Chonavel, C. Karnfelt, A. Peden, F. Bodereau, "Radars FMCW pour l'aide a la conduite automobile".

[13] S. Pechberti, D. Gruyer and V. Vigneron, "Radar simulation in SiVIC platform for transportation issues: Antenna and Propagation channel modelling", ITSC2012, Anchorage, 2012.

[14] F. Gallee, C. Appere, D. Leroux, A. Peden, M. Ney, D. Gruyer, "Development of a simulation plateform for ACC radar application", ITST2011, St Petersburg, Russia, August, 25th. 2011, pp 715-720.

[15] D. Gruyer, S. Glaser, S. Pechberti, R. Gallen, N. Hautiere, "Distributed Simulation Architecture for the Design of Cooperative ADAS", Fastzero, Tokyo, JAPAN, September 5, 2011

[16] D. Gruyer, S. Demmel, B. d'Andrea-Novel, A. Lambert, A. Rakotonirainy, "Simulation architecture for the design of Cooperative Collision Warning systems", ITSC2012, Anchorage, USA, 16-19 Sept. 2012, pp 697-703.

[17] D. Gruyer, V. Berge-Cherfaoui, "Multi-objects association in perception of dynamical situation", UAI'99, Stockholm, Sweden, July 1999. 